\title{
Measurement of the water-Cherenkov detector response to inclined muons using an RPC hodoscope
}

\author{
Pedro Assis $^{* a}{ }^{\dagger}$ for the Pierre Auger Collaboration ${ }^{b}$ \\ ${ }^{a}$ LIP / IST, Lisboa, Portugal \\ ${ }^{b}$ Observatorio Pierre Auger, Av. San Martín Norte 304, 5613 Malargüe, Argentina \\ E-mail: auger_spokespersons@fnal.gov \\ Full author list: http://www.auger.org/archive/authors_2015_06.html
}

\begin{abstract}
The Pierre Auger Observatory operates a hybrid detector composed of a Fluorescence Detector and a Surface Detector array. Water-Cherenkov detectors (WCD) are the building blocks of the array and as such play a key role in the detection of secondary particles at the ground. A good knowledge of the detector response is of paramount importance to lower systematic uncertainties and thus to increase the capability of the experiment in determining the muon content of the extensive air showers with a higher precision. In this work we report on a detailed study of the detector response to single muons as a function of their trajectories in the WCD. A dedicated Resistive Plate Chambers (RPC) hodoscope was built and installed around one of the detectors. The hodoscope is formed by two stand-alone low gas flux segmented RPC detectors with the test water-Cherenkov detector placed in between. The segmentation of the RPC detectors is of the order of $10 \mathrm{~cm}$. The hodoscope is used to trigger and select single muon events in different geometries. The signal recorded in the water-Cherenkov detector and performance estimators were studied as a function of the trajectories of the muons and compared with a dedicated simulation. An agreement at the percent level was found, showing that the simulation correctly describes the tank response.
\end{abstract}

The 34th International Cosmic Ray Conference,

30 July- 6 August, 2015

The Hague, The Netherlands

\footnotetext{
*Speaker.

${ }^{\dagger}$ P. Assis gratefully acknowledges the financial support by FCT/COMPETE/QREN
} 


\section{Introduction}

The water-Cherenkov detector (WCD) is the main building block of the Surface Detector (SD) of the Pierre Auger Observatory. The WCD samples the shower charged particles that arrive at ground [1]. Through the number of particles that reach each SD station and their arrival times it is possible to infer the nature and arrival direction of the primary cosmic ray which initiated the extensive air shower (EAS). Moreover, using sophisticated analyses that explore the WCD response to different shower components, it is also possible to assess the EAS muon content, an important quantity to evaluate our current understanding of the shower description. Naturally, these analyses require a deep understanding of the detectors.

In this work, which extends the measurements performed in Orsay with a test WCD equipped with scintillators [2], we aim to improve the understanding of the parameters governing the light propagation inside the WCD, namely the light reflection and absorption. For that, a hodoscope was mounted, enabling the selection of particular particle trajectories inside the WCD.

The detailed information from these measurements can be used to fine-tune the simulation of the detector and contribute to a reduction of the present systematic uncertainties deriving from the optical properties of the WCD.

\section{Description of the experimental apparatus}

The SD [1] is composed of an array of WCD which are molded plastic containers (tanks) with a sealed liner filled with purified water. The inner side of the liner is surfaced with Tyvek ${ }^{\circledR}$ to reflect the light generated in the water by the Cherenkov effect of the crossing relativistic particles. At its top, three PMTs are installed by means of transparent plastic windows. A detailed description of the WCD can be found in [3]. The Gianni Navarra detector, the focus of this work, is a test WCD located in the Malarguie central campus and thus disconnected from the actual SD.

Resistive Plate Chambers (RPCs) are gaseous detectors capable of achieving very high detection efficiency and high timing accuracy. The RPCs are made of a sensitive gas volume, where the primary ionization and avalanche multiplication occurs, and by a signal pickup module. The pickup is segmented into 64 pads $\left(\sim 15 \times 19 \mathrm{~cm}^{2}\right.$ each $)$, allowing the estimation of the active region. A review of RPCs and their principle of operation can be found in $[4,5]$. The detectors used in the hodoscope are a result of a specific development of RPCs for the Pierre Auger Observatory $[6,7]$ that was focused on the operation of autonomous stations equipped with RPCs. As such it was necessary to develop RPCs with low gas consumption and simple electronics to meet stringent requirements on price and power consumption.

The hodoscope is formed by two stand alone low gas flux RPC detectors with the testing WCD placed in between. A photograph of the setup is shown in fig. 1. The readout of the RPCs is segmented into small pads, which allows for an accurate reconstruction of the individual muon trajectories. The hodoscope is used to trigger and select single trajectory events in different geometries. Both RPCs are installed in moveable structures allowing for different configurations and hence probing different regions of angles of incidence. 


\section{Data Acquisition system}

The data acquisition (DAQ) system of the setup has two main components: the WCD DAQ and the hodoscope DAQ. The WCD DAQ uses the standard Surface Detector Electronics (SDE) [8]. The Gianni Navarra station is not included in the standard array and is, as such, not connected to the Central Data Acquisition System. Instead, the control and readout of the electronics is performed through a direct link to the console. The largest part of the event data consists of the traces of the three photomultipliers. Occasionally the station records calibration histograms that are computed internally using a dedicated self-trigger. These calibration data consist in the set of the distributions of the baseline and of the charge amplitude of single particles.

The hodoscope electronics is based on a prototype discrete electronics system (PREC) developed for the readout of the RPCs. The system uses an architecture with front-end boards and one motherboard. In the Front End (FE), the signal from each pad is amplified and then a simple threshold is applied to perform a 1-bit digitization. These signals are then fed into a purely digital motherboard comprising 14 FPGAs (Field-Programmable Gate Array) organized in a mother-daughters configuration. The communication between the mother FPGA and a PC is accomplished with an USB connection. For each acquired pad this scheme allows us to record whether there was a signal above the imposed threshold in a window of $1 \mu$ s before the WCD trigger.

Two types of trigger systems are used: the WCD-based trigger and the hodoscope-based trigger. The WCD-based trigger uses the SDE triggering system lowering the threshold to 0.2 VEM to catch signals as low as possible. A VEM is defined as the most probable value for the signal from a center going vertical muon. This SDE trigger was then fed to the PREC system to record the data from the hodoscope. Data synchronization was achieved by means of a global identifier generated by the WCD electronics and communicated to the PREC using auxiliary digital data lines. Upon a trigger, the PREC electronics saves the data for all the pads.

To increase the rate of events with particles crossing both the hodoscope and the WCD, a new triggering scheme was set up, based on the hodoscope signals and implemented in the motherboard. In short, one FPGA from the motherboard looks for a pattern where at least one pad on the top RPC and at least one pad on the bottom RPC were activated in a time window of $40 \mathrm{~ns}$. The generated trigger is then sent internally into all FPGAs of the motherboard and also to the WCD, causing it to acquire the trace of the PMTs and to generate the event identifier.

\section{Dedicated simulation}

To assess the results obtained with the hodoscope, a dedicated simulation was developed. This simulation tries to reproduce the conditions at which the measurements were performed, accounting for geometrical and detector efficiencies.

In order to use a realistic set of events, CORSIKA simulations of the atmospheric particles were run for the Malargüe site. The expected primary cosmic ray fluxes in the range energy $\log (E / \mathrm{eV}) \in[11,15]$ and an isotropic arrival direction were considered in the simulation.

The resulting energy distribution is shown in fig. 1. The all-particle distribution is displayed by the black curve while the different components of the distribution are shown by the filled his- 
tograms. It can be seen that the low energy region is dominated by photons while above a few $\mathrm{GeV}$, only muons contribute.
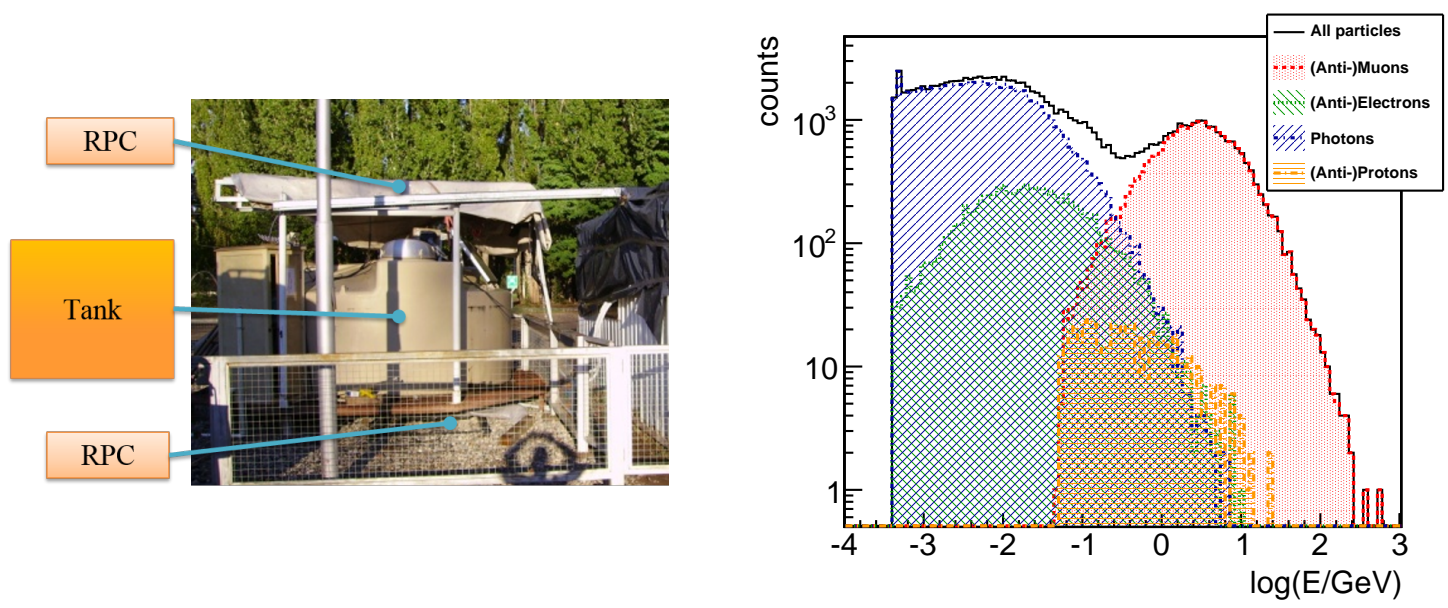

Figure 1: Left: photograph of the muon hodoscope setup at the Gianni Navarra WCD. Right: atmospheric particle energy spectrum used to produce the dedicated simulation.

The simulated atmospheric particles are then injected into a simulation of the WCD+RPC setup using GEANT4. A detailed description of the RPC structure was implemented by taking advantage of the GEANT4 capabilities to describe complex geometries and the physical properties of the materials (fig. 2).
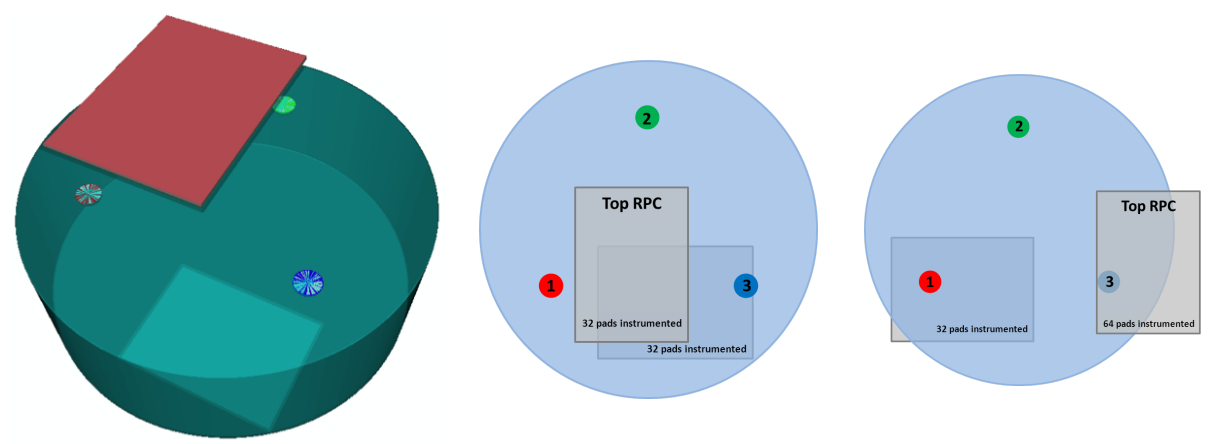

Figure 2: Left: view of one of the RPC configurations as implemented in GEANT4. Middle and right: top view of configuration 1 and 2 respectively (see text for details).

The particle tracking in the WCD, the Cherenkov light generation and propagation is performed exactly in the same way as in the standard simulation of the WCD in the Auger $\overline{\text { Offline }}$ software [9]. The tracking in the RPCs enables the recording of the incoming particle position, direction and the time, as well as the ionizing energy deposited in the gas, which is then used to generate charge pulses. The electronic signals are then effectively simulated by a parameterisation.

\section{Acquired Data and Analysis}

The data presented in this work correspond to three experimental campaigns: in campaign 1 the WCD trigger was used and the RPC were almost on top of one another allowing to study zenith 
angles in $\sim\left[0^{\circ}, 40^{\circ}\right]$; in campaign 2 the RPCs were moved to cover zenith angles in $\sim\left[20^{\circ}, 55^{\circ}\right]$; in campaign 3 the later RPC configuration was kept and the hodoscope-based trigger was used. The number of collected events in each campaign was, respectively, $1.2 \times 10^{3}, 2.3 \times 10^{3}$ and $3.5 \times 10^{5}$.

The RPC data were analyzed with the goal of determining the trajectory of single atmospheric muons passing through the hodoscope by reconstructing the muon zenith and azimuth angles, as well as the muon tracklength $(L)$ inside the WCD water. A three-step analysis was developed for this purpose.

Firstly, noisy and dead RPC pads were identified and removed from the analysis. In fig. 3 the selected pads for the data analysis, for the third data collection campaign, are shown.
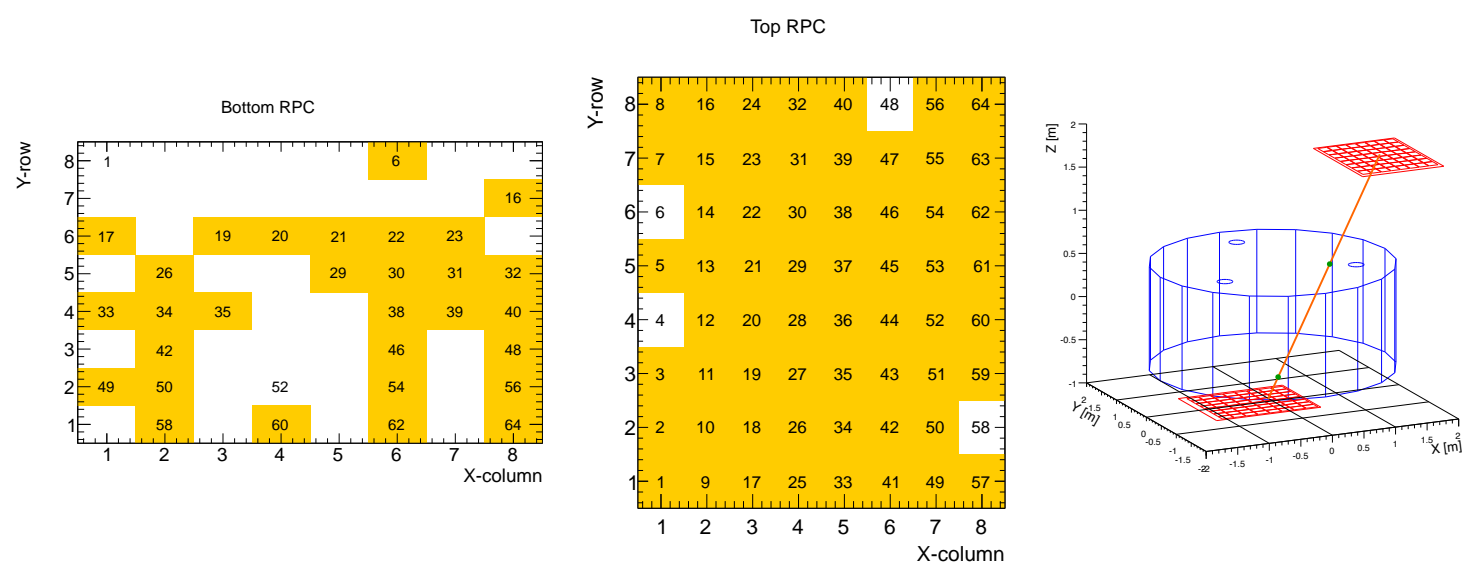

Figure 3: Left and middle: scheme of the bottom and top RPC pads in experimental campaign 3. The pads with readout instrumentation are numbered and those that passed the selection cuts are shown in orange. Right: schematic view of a muon track going through the hodoscope mounted on the Gianni Navarra WCD.

The second step of the data analysis is the event selection. Since the goal of this work is to study the WCD response as a function of the muon trajectory, the interesting events are those in which a single muon triggers the WCD and both RPCs. The event selection criteria are therefore to require one and only one hit in the top RPC and one and only one hit in the bottom RPC. This defines the "single-hit" selection. The contamination of the sample by random coincidences was studied and found to be only of a few percent, thus having a negligible impact on the results.

In the third step the muon trajectory, shown schematically in fig. 3 (right), is reconstructed from the positions of the hit pads.

The direction of the traversing particles is calculated from the center of the two activated pads. Since each pad has a finite area $\left(\sim 15 \times 19 \mathrm{~cm}^{2}\right)$ an uncertainty is introduced in the estimation of the geometry which is the solid angle subtended by the two pads. The resulting hodoscope resolution in terms of tracklength, zenith angle and azimuth angle is of the order of a centimeter, $1^{\circ}$ and $2^{\circ}$, respectively for near vertical trajectories.

Finally, the baseline, peak time-bin, current and charge for high gain traces of individual PMTs and for the total trace were estimated using standard analysis methods [10] of the Pierre Auger Collaboration. These quantities were studied as a function of the muon trajectory.

The WCD calibration data were used to make the conversion from charge (in integrated ADC counts) to signal in VEM units. The muon peak was fitted by adjusting Gaussian functions to the 
calibration data to determine the position of the maximum and therefore to obtain the calibration constants, relating the charge in integrated ADC counts with the maximum of the muon peak that corresponds to 1.04 VEM [10]. While in campaigns 1 and 2 the calibration histograms were available and the above mentioned calibration procedure was followed, in campaign 3 they were not. In this case, the data were rescaled to the simulation value for $L \in[1.25,1.3] \mathrm{m}$.

\section{Preliminary Results}

The measured total signal distribution (charge distribution) upon applying the VEM calibration procedure is shown in fig. 4 (left). The red histogram is the signal distribution for all recorded events while the black one is the distribution after the single-hit selection cut.
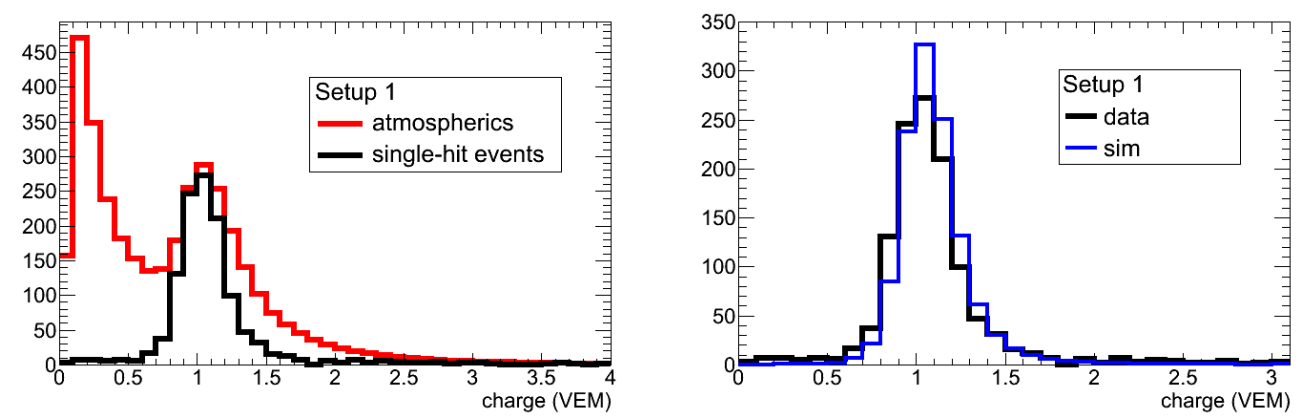

Figure 4: Left: charge distribution in data, before and after selecting single hit events; Right: charge distributions for single hit events in data and simulation. The number of simulated events was normalized to the number of acquired events.

As a consequence of this cut, the electromagnetic component of the atmospheric particle flux is severely reduced. This leads to the disappearance of the first, low energy, peak shown in this plot, confirming the selection of muons. The events with high charge are characterized by having large tracklengths or/and high multiplicity. As the RPCs configuration limits the maximum tracklength, and the required multiplicity of the cut is 1 , the tail at the right of this plot is also reduced.

In fig. 4 (right), a comparison between data and simulation is shown for the total signal distribution requiring single hit events. It can be seen that the histograms have a good qualitative agreement with both peaks close to 1 VEM.

Further insight on the details of the WCD response can be gained by studying the dependency of the signal as a function of the muon tracklength $(L)$ in the WCD. Fig. 5 shows the average trace for different ranges of $L$, showing as expected an increase of the signal amplitude with tracklength.

In fig. 6 the dependency of the signal with $L$ is shown. Data are plotted in black and simulation in blue. As expected, the signal increases as a function of the tracklength in the WCD. The experimental data are in agreement with the simulation to the few percent level. Moreover, the geometry of the selected tracks introduces some structures that are well described by the simulation.

\section{Summary and Prospects}

In this work, we reported on the hodoscope system installed at the Gianni Navarra WCD for the detailed study of the detector response to individual muons traversing the WCD. We focus on 


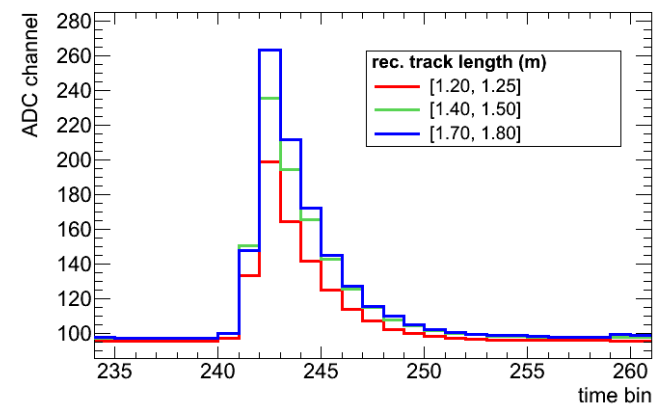

Figure 5: Average trace (PMTs 2 and 3) for a few selected tracklength intervals.

the setup, the simulation and the results from the first collected data. The system is comprised of a hodoscope realized with RPCs that is able to tag single muon events and to estimate the trajectory, namely the tracklength in the WCD, with an accuracy of a few $\mathrm{cm}$. A dedicated simulation was developed to take into account not only the geometry of the setup but also a realistic flux of atmospheric particles. The signal evolution with the tracklength behaves as expected when compared to the simulation. A maximum deviation of $\sim 2 \%$ was found for the acquired data, showing that the simulation correctly describes the tank response.

In the future we foresee making several upgrades to the setup in order to stabilize and enhance its acquisition capabilities. The most important upgrade will be a change in the mechanical setup that will allow us to rotate the RPCs and increase the limit in the zenith angle up to almost $90^{\circ}$.

\section{References}

[1] Pierre Auger Collaboration, A. Aab et al., The Pierre Auger Cosmic Ray Observatory, accepted for publication in Nucl. Instrum. Meth. A (2015) [arXiv:1502.0132].

[2] Pierre Auger Collaboration, M. Aglietta et al., Response of the Pierre Auger Observatory Water Cherenkov Detectors to Muons, in Proc. 29th ICRC, 2005.

[3] Pierre Auger Collaboration, I. Allekotte et al., The surface detector system of the Pierre Auger Observatory, Nucl. Instrum. Meth. A 586 (2008) 409-420.

[4] P. Fonte, Applications and new developments in RPCs, IEEE Trans. Nucl. Sci. 49 (2002) 881-887.

[5] P. Fonte, Review of RPC simulation and modelling, PoS RPC2012 (2012) 033.

[6] L. Lopes, P. Fonte, and M. Pimenta, Study of standalone RPC detectors for cosmic ray experiments in outdoor environment, JINST 8 (2013) T03004.

[7] L. Lopes, P. Assis, A. Blanco, M. Cerda, N. Carolino, et al., Resistive Plate Chambers for the Pierre Auger array upgrade, JINST 9 (2014) C10023.

[8] Pierre Auger Collaboration, T. Suomijärvi et al., Surface detector electronics for the Auger Observatory, Proc. 27th ICRC (2001) 756-759.

[9] S. Argirò et al., The offline software framework of the Pierre Auger Observatory, Nucl. Instrum. Meth. A 580 (2007) 1485-1496. 

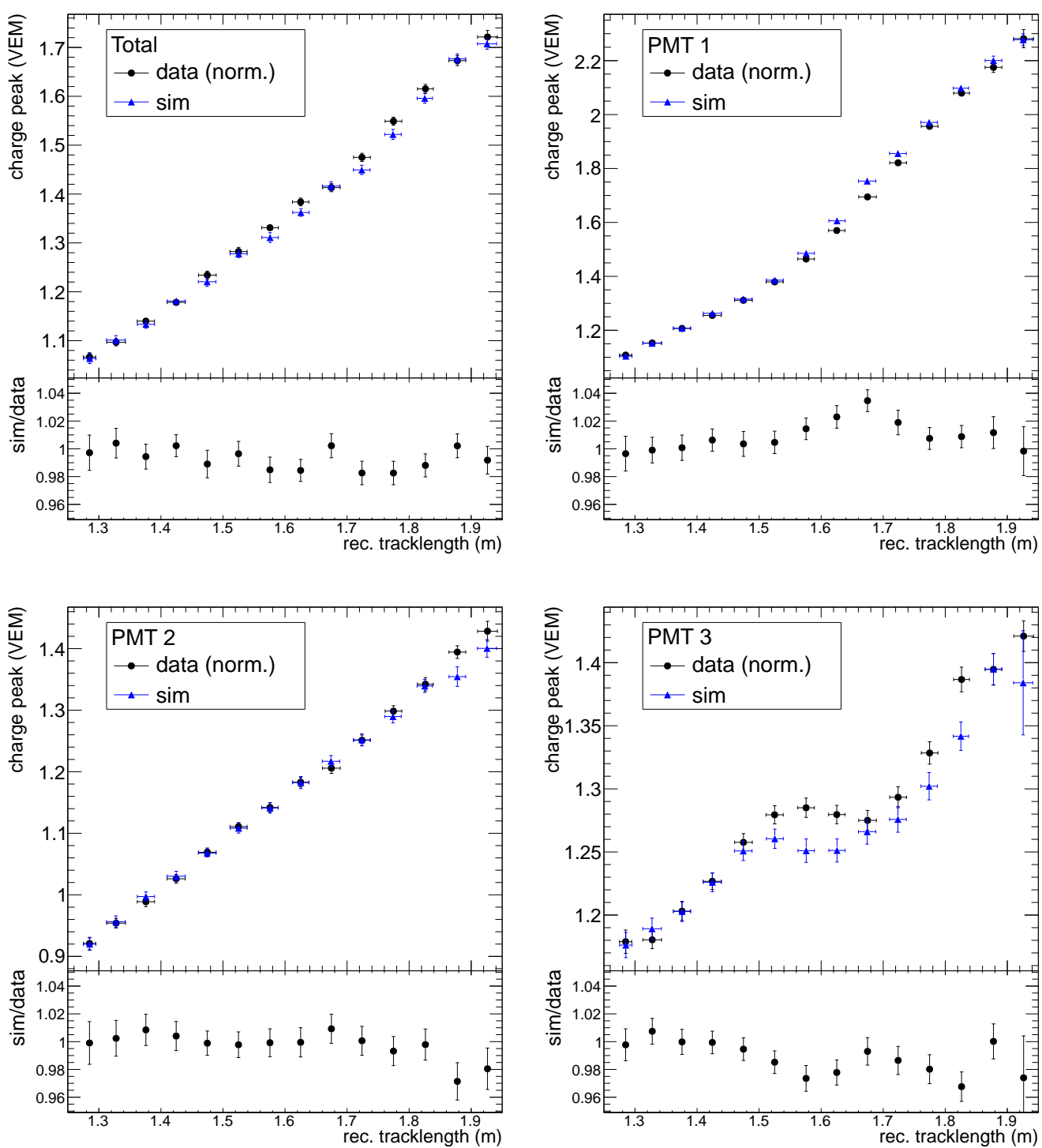

Figure 6: Peak of the charge distribution as a function of the muon tracklength in the WCD water, for the total PMT signal (top left) and for individual PMTs in campaign 3. Data were rescaled to the simulation value for $L \in[1.25,1.3] \mathrm{m}$. The ratio between simulation and data is also shown at the bottom.

[10] Pierre Auger Collaboration, X. Bertou et al., Calibration of the surface array of the Pierre Auger Observatory, Nucl. Instrum. Meth. A 568 (2006) 839-846. 